\title{
Surgical site infection prevention: An analysis of compliance with good practice in a teaching hospital
}

Journal of Infection Prevention 20I7, Vol. 18(6) 30I-306 (C) The Author(s) 2017 Reprints and permissions: sagepub.co.uk/journalsPermissions.nav DOI: 10.1 |77/|757|77417703190 jip.sagepub.com

\section{Adriana Cristina de Oliveira ${ }^{1,2}$ and Camila Sarmento Gama ${ }^{2}$}

\begin{abstract}
Background: Surgical teams play a critical role in reducing surgery-related risks during preoperative and intraoperative phases.
\end{abstract}

Aim: To analyse the preoperative and intraoperative practices adopted by surgical teams in surgical site infections prevention.

Methods: This was a cross-sectional study conducted during April-September 2013 in a large university hospital in Belo Horizonte, Minas Gerais, Brazil. It was conducted through observation of the surgical practice during preoperative and intraoperative phases of procedures used by a gastroenterological, cardiovascular and paediatric surgical team.

Results: A total of 100 surgeries were monitored. Hair removal was performed for $20 \%$ of the patients inside the operating room by professionals using clippers in $65 \%$ of operations. The antimicrobial agent of choice was appropriate/ satisfactory in $62 \%$ of the operations and administered up to 60 min before surgical incision in $90.3 \%$ of the cases. The operating room door was kept closed in $4 \%$ of these procedures.

Discussion: Some preoperative measures for surgical site infection prevention were not adhered to by the professionals who were monitored in this study. It is recommended that surgical teams undergo professional surveillance and training to highlight the necessity and importance of implementing measures to improve the quality of care provided to surgical patients.

Keywords

Surgical wound infection, operating rooms, patient safety

Date received: 8 September 2016; accepted: 12 March 2017

\section{Background}

Despite advances in surgical practice, surgical site infections (SSI) are frequent complications in patients who undergo surgery (Anderson et al., 2014). In Brazil, 14-16\% of patients are hospitalised because of SSIs (National Health Surveillance Agency, 2009). According to the European Centre for Disease and Prevention Control (ECDC), in 2011-2012 SSI accounted for $19.6 \%$ of healthcare-associated infections (HAI) (ECDC, 2013).

SSIs can double the length of stay in hospital and thereby increase the costs of healthcare. Additional costs attributable to SSI in the range of £814-6626 per infection have been reported as well as physical, emotional and financial damages to the affected patients (National Institute for
Health and Clinical Excellence [NICE], 2008; World Health Organization [WHO], 2009b).

Although SSIs often have multifactorial aetiology, surgical teams play a critical role in reducing surgery-related

\footnotetext{
'School of Nursing, Universidade Federal de Minas Gerais, Belo Horizonte-Minas Gerais, Brazil

${ }^{2}$ Center for Studies and Research in Infection-Related Health Care (NEPIRCS)/CNPq, Belo Horizonte-Minas Gerais, Brazil
}

Corresponding author:

Adriana Cristina de Oliveira, Avenue Professor Alfredo Balena 190, Santa Efigênia 30.130-100, Belo Horizonte-Minas Gerais, Brazil.

Email: adrianacoliveira@gmail.com 
risks during preoperative and intraoperative phases (Alexander et al., 2011).

Preventive measures, such as the proper use of surgical attire, the removal of hand jewellery by professionals present in the operating room (OR) prior to surgery, hair removal with clippers only when necessary, minimising the unnecessary traffic during surgery, keeping the OR door closed during surgery and having patients take preoperative showers, are recommended to control the occurrence of SSIs (Anderson et al., 2014; NICE, 2013; WHO, 2016). These measures contribute to the reduction of microbial load and therefore minimise the transfer of microorganisms to the site of surgical incision (Alexander et al., 2011; NICE, 2008).

Although the importance of these measures is well described in the literature and in national and international manuals, there are few studies describing the routine implementation of these measures during surgical procedures (Durando et al., 2012).

This research therefore aims to investigate how well practices recommended to prevent surgical site infection in the OR are implemented at a public university hospital in Belo Horizonte, Minas Gerais, Brazil.

Providing information on lapses in best practice to nurses and other professionals involved in surgical care may enable planning of interventions to improve the safety and quality of care for the surgical patient.

\section{Methods}

A cross-sectional study was conducted at a large hospital of a public university in Belo Horizonte between April and September 2013, following approval by the Research Ethics Committee of the Federal University of Minas Gerais (ETIC 114116512.1.0000.5149).

Data collection was performed according to the schedule of routine surgical procedures carried out in the departments of gastrointestinal surgery, cardiovascular surgery and paediatric surgery. These surgical specialties were chosen because they represent the highest surgical volume in the institution.

Three researchers, who were not known by the surgical teams, participated in data collection as part of a broader study to assess glove perforation used by surgeons during the procedure. Data on preoperative shower, patient American Society of Anesthesiologists (ASA) classification and potential contamination during the surgical procedure were obtained from the medical records.

Data on antimicrobial administration, hair removal (method, place and time), wearing of surgical attire and hand jewellery, number of people inside the room, length of time that the OR door remained open and length of surgical procedure were collected by the researcher in the OR for the duration of the procedure.

Every person present in the OR for more than $5 \mathrm{~min}$ from the time of surgical incision to the time of its closing were observed.
The time during which the door was kept open or closed was measured after the surgical incision was made until its closure; the time that door was left open during the passage of persons and equipment in and out of the OR was not considered.

The data were entered in a survey form developed for this purpose.

The definition of a surgical procedure was 'surgery performed on an inpatient or an outpatient that takes place during a single trip to the OR where at least one incision is made through the skin or mucous membrane, including the use of a laparoscopic approach, and the incision is primarily closed before the patient leaves the OR' (National Healthcare Safety Network [NHSN], 2013).

Informed consent was obtained from staff who were observed.

The collected data were entered and analysed by using the statistical software Statistical Package for Social Sciences (SPSS ${ }^{\circ}$ ) version 20.0; descriptive analysis was used for variables studied, including percentages, measures of central tendency and dispersion.

\section{Results}

\section{General characteristics of the sample}

A total of 100 surgical procedures performed by 116 participants (medical practitioners and other staff) were monitored. Most participants were men and the mean age was 33.9 years (age range, 23-77 years). In the group of participants from the surgical teams, there were 36 preceptors (medical professors) (31\%), 41 medical residents (35.3\%), 27 medical students $(23.3 \%), 11$ nursing technicians $(9.5 \%)$, and one researcher $(0.9 \%)$.

The duration of the surgery was up to $149 \mathrm{~min}$ in 65 procedures $(65 \%)$ and $\geq 150$ min for 35 procedures $(35 \%)$; the shortest procedure was $30 \mathrm{~min}$ and the longest procedure lasted $419 \mathrm{~min}$.

The distribution of procedures according to medical specialty was as follows: 62 gastrointestinal procedures $(62 \%)$; 20 paediatric procedures $(20 \%)$; and 18 cardiovascular procedures $(18 \%)$.

\section{Preoperative procedures}

Hair removal was performed for 20 patients $(20 \%)$ in the OR (Table 1). It is noteworthy that two patients arrived in the OR with hair already removed from the area of the incision. One patient had used a depilatory cream eight days before surgery and the other shaved the incision area with a razor three days before surgery. The remaining patients had their operation in a hairless area and therefore it was not necessary to remove it.

Regarding the preoperative shower on the day of surgery, 91 patients $(91 \%)$ showered and one patient (1\%) did not. This information was not available for eight patients 
Table I. Characterization of hair removal and preoperative shower in surgical patients observed in this study.

\begin{tabular}{|lcc|}
\hline Variable & $\mathrm{n}$ & $\%$ \\
\hline Method used for hair removal & 20 & 100 \\
\hline Razor & 7 & 35 \\
\hline Clipper & 13 & 65 \\
\hline Timing of hair removal & 20 & 100 \\
\hline Up to 60 minutes before surgical incision & 20 & 100 \\
\hline Place of preoperative shower & 91 & 100 \\
\hline Hospital & 54 & 59.3 \\
\hline Home & 37 & 40.7 \\
\hline $\begin{array}{l}\text { Antiseptic solution use during preoperative } \\
\text { shower }\end{array}$ & 91 & 100 \\
\hline Yes & & \\
\hline No & 2 & 2.2 \\
\hline
\end{tabular}

$(8 \%)$ who had no description of the procedure in their medical records or in any other source of information. It is noteworthy that only two patients $(2.2 \%)$ reported taking a preoperative shower with an antiseptic solution. One patient used chlorhexidine and the other patient did not describe the type of antiseptic that was used. Both patients were undergoing mitral valve and aortic procedures (Table 1).

To confirm whether the appropriate antimicrobial agent was chosen for each procedure monitored, a recommendation guide published by the Commission of Infection Control from the institution where the study was conducted (Committee on Hospital Infection Control, 2012) was consulted, combined with a discussion with an institutional expert in the use of antimicrobial agent during surgery. However, when surgical procedures were not included in the institution guide or there was doubt regarding the appropriate choice of antimicrobial agent to be used, the manual of the American Society of Health-System Pharmacists (ASHP) (Bratzler et al., 2013) was consulted. Each case was analysed individually.

The observed distribution of antimicrobial agents according to the recommendations proposed is summarised in Table 2 .

In a total of 68 operations, the administration of antimicrobials was considered suitable/acceptable; of these, antimicrobials were required and used in 62 operations whereas six operations did not require or use an antimicrobial agent.

Among the procedures during which an antimicrobial agent was required and used, the time of administration was more than 60 min before the incision was made in two cases $(3.2 \%), 0-60 \mathrm{~min}$ before the incision in 56 cases
$(90.3 \%)$ and more than $10 \mathrm{~min}$ after the incision in four cases $(6.5 \%)$, excluding patients where antimicrobial agents were being prescribed for therapeutic purposes.

\section{Intraoperative procedures}

With regard to the door of the OR remaining closed throughout the operation, this was observed in only four operations (4\%). The door was kept open during half of the surgical time in three of the procedures (3\%), up to $90 \%$ of the time during six procedures $(6 \%)$ and throughout the operation time for 87 of the procedures $(87 \%)$.

The mean number of staff members with a specific function in the OR was 6.5 and the mean number of individuals who were present during the surgical procedures was 8.1 . Only 29 surgeries $(29 \%)$ had the exact number of individuals who were required to perform functions during the surgery.

Finally, the use of proper safety attire and personal protective equipment (PPE), as well as hand jewellery worn by members of the surgical team who were in contact with the surgical field (surgeon, assistant and scrub nurse), were evaluated. Of the total number of individuals of the surgical team $(\mathrm{n}=353$, because the same person was observed during different procedures), 95 (26.9\%) wore goggles, 202 $(57.2 \%)$ wore shoe covers, $69(19.5 \%)$ wore a cap that properly covered their hair and ears, $352(99.7 \%)$ wore a surgical gown, $338(95.8 \%)$ wore a surgical mask that was positioned properly and $353(100.0 \%)$ wore surgical gloves.

It was observed that a surgical team assistant was wearing a ring during the procedures. Other individuals apart from surgical teams $(n=467)$ in the OR were also evaluated during the operation regarding their use of attire and hand jewellery. These individuals were anaesthesiology residents, anaesthesiology preceptors, observers (residents, medical and nursing students, nursing technicians), endoscopists, perfusionists and radiology technicians.

Overall, these professionals did not use goggles, gowns or surgical gloves, because it was not necessary, with the exception of some anaesthetists and preceptors who wore gowns and surgical gloves when performing aseptic punctures.

Regarding the use of other PPE, of the observed 467 professionals who were circulating outside the sterile field, $98(21 \%)$ used a surgical cap properly and $188(40.3 \%)$ used a surgical mask that was positioned correctly. With regard to hand jewellery, of these participants, $109(23.3 \%)$ wore a ring, $20(4.3 \%)$ wore bracelets and $150(32.1 \%)$ wore a watch.

\section{Discussion}

Some proposed preoperative measures for SSI prevention were not adhered to by the professionals who were monitored in this study. With regard to hair removal, it 
Table 2. The distribution of antimicrobial use according to the recommendations by the Commission of Infection Control and the ASHP combined with the discussion with an expert in the area of antimicrobials auditing of the institution.

\begin{tabular}{|lllll} 
& \multicolumn{3}{c}{ Use of antimicrobial agent } & \\
\cline { 2 - 5 } Classification of antimicrobial prophylaxis & Yes $(\%)$ & No $(\%)$ & Therapeutic use prior to surgery (\%) & Total \\
\hline Appropriate & 48 & 6 & 0 & 54 \\
\hline Inappropriate & 18 & 7 & 0 & 25 \\
\hline Satisfactory* & 14 & 0 & 0 & 14 \\
\hline Not applicable & 0 & 0 & 7 & 7 \\
\hline Total & 80 & 13 & 7 & 100 \\
\hline
\end{tabular}

*Refers to the choice of a substitute antimicrobial agent that is able to target microorganisms potentially present in the surgical wound if the agent of first choice was not selected, in accordance with local protocols.

is recommended that, in accordance with national and international guidelines, patients should not have their hair removed unless the presence of the hair interferes with the surgery. If necessary, the use of electric clippers to remove hair from the incision site is recommended immediately prior to surgery (NICE, 2013; WHO, 2016).

Although some literature advises hair removal outside the OR (Anderson et al., 2014), the WHO does not make any recommendation about hair removal location, as no studies evaluated the effect of settings where hair removal is performed (OR versus ward or home) with the outcome of SSI (WHO, 2016). In this study, hair removal was done inside the OR; this was in consonance with the literature from that period, which did not mention where hair removal should take place (NICE, 2008, 2013).

The findings from this study indicated a high level of compliance with this recommendation as the majority of patients had their hair removed with electric clippers prior to surgery (Castella et al., 2006; Durando et al., 2012).

A preoperative shower is widely recommended (NICE, 2008, 2013; WHO, 2016). Taking such a shower at least the night before surgery has been recommended by the WHO. NICE recommends that the patient showers either the day before or the day of surgery. The use of antimicrobial soap or plain soap is still inconclusive in literature; therefore, no organisation advises one over the other (NICE, 2008, 2013; WHO, 2016).

In the present study, as in other studies (Castella et al., 2006; Durando et al., 2012), patients reported taking a shower at the hospital on the day of surgery, without using antiseptic solution, which follows the recommendations worldwide (NICE, 2008, 2013; WHO, 2016).

The available scientific evidence is controversial in showing a correlation between the use of antiseptic solution and a reduction in SSI (Anderson et al., 2014). Although the use of antiseptic solution cannot be directly related to the reduction in SSIs, their use should be evaluated regarding their role in reducing microbial load (Edmiston Jr et al., 2015). Considering that most contamination occurs via microorganisms present on the patient's skin, a preoperative shower can potentially reduce the risk of endogenous infection (NICE, 2008).

Surgical antimicrobial prophylaxis is one of the most important methods available to prevent SSIs (Alexander et al., 2011). These agents reduce the microbial load at the surgical site during surgery (WHO, 2016).

However, the use of antimicrobial prophylaxis is not recommended for all surgical interventions because of the high costs involved, potential adverse events and the possibility of antibiotic resistance and colitis caused by Clostridium difficile (Alexander et al., 2011).

Our study found that appropriate antimicrobial agent selection occurred for only $62 \%$ of surgical procedures. This is of concern because incorrect prescriptions or lack of appropriate antibiotics can result in unnecessary expenses and the development of bacterial resistance or SSIs (Alexander et al., 2011). Variation in rate of compliance has been reported by other studies. For example, in Italy, 55.2\% of antimicrobial choices were found to be inappropriate (Durando et al., 2012), whereas in an Australian study, there was a high rate of appropriate choice of antibiotics for cardiac and orthopaedic surgeries (97\%) and for colorectal surgeries (89\%) (Friedman et al., 2013).

The recommended time administration for most antimicrobial agents is within 60 min before surgery, except vancomycin and fluoroquinolones: where $120 \mathrm{~min}$ before surgery is recommended (Anderson et al., 2014; Bratzler et al., 2013). This is required to ensure ideal serum and tissue concentrations at the time of incision and to minimise the possibility of infection (Bratzler et al., 2013; WHO, 2009a, 2016). The results from this study show a good compliance with the time recommended to antimicrobial administration as $90.3 \%$ gave the antimicrobial agents to patients up to $60 \mathrm{~min}$ before the operation.

Keeping the OR door closed during the surgical procedure except during the passage of equipment/materials and professionals into the room is important to avoid OR air contamination and unnecessary traffic (Andersson et al., 
2012; DeBaun, 2016). It was only accomplished in $4 \%$ of the operations monitored. In similar studies (Castella et al., 2006; Durando et al., 2012), the doors were kept open for the majority of the duration of the procedure, demonstrating the need for review of this requirement during surgical procedures to ensure compliance with closed doors.

It was observed that the number of individuals present in the OR during the surgeries was slightly higher than the number reported in the literature (Castella et al., 2006; Durando et al., 2012). Although limiting the number of people present and traffic during a procedure is recommended in order to minimise air contamination, distractions and reduced productivity which can contribute to SSIs (Anderson et al., 2014; Andersson et al., 2012; DeBaun, 2016), the observations from this study can be partially explained by the fact that the study was conducted at a teaching hospital where students usually accompany surgical teams and learn by observing the procedure.

Regarding hand jewellery, only an assistant from the surgical team who was properly attired wore a ring. Wearing hand jewellery on the hands and forearms is not recommended by national and international institutions (National Health Surveillance Agency, 2007; NICE, 2013; WHO, 2009a). Also, it is not recommended in the institution where the study was carried out.

Although there is no evidence that wearing jewellery contributes to an increased rate of SSIs, this practice should not be encouraged, as studies have shown increased microbial colonisation in areas covered by hand jewellery owing to lack of contact of antiseptic at these sites (WHO, 2009a). Moreover, potential injury to the user or patients may occur owing to an increased risk of punctures and tears in gloves caused by the wearing of such hand jewellery (Castella et al., 2006).

Regarding the use of attire by surgical team members who were in contact with the surgical field, it was noted that compliance was satisfactory in the use of surgical gowns, masks (covering mouth and nose) and gloves. Adherence in the proper use of hats, shoe covers and goggles was inadequate.

The use of PPE helps to minimise direct contact with the wound, as well as protection from biological material that may contaminate either the patient or surgical team members (NICE, 2008; WHO, 2009a).

The observed failure to adhere to these practices may indicate negligence or lack of knowledge on the part of these professionals and should be corrected.

This research has some limitations, such as no postoperative follow-up of surgical patients to evaluate the association between non-compliance with infection prevention measures and the outcome SSI. The number of operations included in the study was also limited by the time available to collect data.

Although this study did not address all the recommendations for the prevention and control of SSIs, such as ventilation, cleaning and disinfection of environmental surfaces, sterilisation of surgical instruments, asepsis and preoperative skin preparation and preoperative hand/forearm antisepsis of the surgical team, it identified aspects of local practice in need of attention, review and improvement through clear infection control policies. This approach may be of value in other surgical centres.

The results suggest that healthcare workers in the surgical centre would benefit from training to highlight the importance of implementing these measures in everyday practice and improve the quality of care provided to surgical patients. Moreover, audit and feedback to employees regarding the implementation of policy recommendations can contribute increasing compliance.

\section{Declaration of conflicting interests}

The author(s) declared no potential conflicts of interest with respect to the research, authorship, and/or publication of this article.

\section{Funding}

The author(s) disclosed receipt of the following financial support for the research, authorship, and/or publication of this article: This study was supported by the Pró-Reitoria de Pesquisa of the Universidade Federal de Minas Gerais.

\section{Peer review statement}

Not commissioned; blind peer-reviewed.

\section{References}

Alexander JW, Solomkin JS and Edwards MJ. (2011) Updated recommendations for control of surgical site infection. Annals of Surgery 253: 1082-1093.

Anderson DJ, Podgorny K, Berríos-Torres SI, Bratzler DW, Dellinger EP, Greene L, Nyquist AC, Saiman L, Yokoe DS, Maragakis LL and Kaye KS. (2014) Strategies to prevent surgical site infections in acute care hospitals: 2014 update. Infection Control and Hospital Epidemiology 35: 605-627.

Andersson AE, Bergh I, Karlsson J, Eriksson BI and Nilsson K. (2012) Traffic flow in the operating room: An explorative and descriptive study on air quality during orthopedic trauma implant surgery. American Journal of Infection Control 40: 750-755.

Bratzler DW, Dellinger P, Olsen KM, Perl TM, Auwaerter PG, Bolon MK, Fish DN, Napolitano LM, Sawyer RG, Slain D, Steinberg JP and Weinstein RA. (2013) Clinical practice guidelines for antimicrobial prophylaxis in surgery. American Journal of Health-System Pharmacy 70: 195-283.

Castella A, Charrier L, Legami VD, Pastorino F, Farina EC, Argentero PA, Zotti CM and the Piemonte Nosocomial Infection Study Group. (2006) Surgical site infection surveillance: analysis of adherence to recommendations for routine infection control practices. Infection Control and Hospital Epidemiology 27: 835-840.

Committee on Hospital Infection Control. (2012) Guide antimicrobial use and recommendations for prevention of healthcare associated infections. 2nd ed. Belo Horizonte: Hospital das Clínicas of Universidade Federal de Minas Gerais.

DeBaun B. (2016) Looking forward - infection prevention in 2017. AORN Journal 104: 531-535.

Durando P, Bassetti M, Orengo G, Crimi P, Battistini A, Bellina DD, Talamini A, Tiberio G, Alicino C, Iudici R, Sticchi C, Ansaldi F, 
Rossi A, Rosso R, Viscoli C, Icardi G and the surgical audit team of the San Martino University Hospital of Genoa. (2012) Adherence to international and national recommendations for the prevention of surgical site infections in Italy: Results from an observational prospective study in elective surgery. American Journal of Infection Control 40: 969-972.

Edmiston CE Jr, Lee CJ, Krepel CJ, Spencer M, Leaper D, Brown KR, Lewis BD, Rossi PJ, Malinowski MJ and Seabrook GR. (2015) Evidence for a standardized preadmission showering regimen to achieve maximal antiseptic skin surface concentrations of chlorhexidine gluconate, $4 \%$, in surgical patients. JAMA Surgery 150: 1027-1033.

European Centre for Disease Prevention and Control. (2013) ECDC Surveillance Report: Point prevalence survey of healthcare-associated infections and antimicrobial use in European acute care hospitals 2011-2012. Stockholm: ECDC.

Friedman ND, Styles K, Gray AM, Low J and Athan E. (2013) Compliance with surgical antibiotic prophylaxis at an Australian teaching hospital. American Journal of Infection Control 41: 71-74.
National Health Surveillance Agency. (2007) Manual of Patient Safety: Hand Hygiene. Brasília: Health Ministry.

National Health Surveillance Agency. (2009) Surgical site. National criteria of healthcare associated infections. Brasília: Health Ministry.

National Healthcare Safety Network (2013). NHSN Protocol Clarification. $9 p$. Available at: www.cdc.gov/nhsn/pdfs/validation/2013/2013-pscmanual-validate.pdf (accessed 2 April 2017)

National Institute for Health and Clinical Excellence. (2008) Surgical Site Infection: prevention and treatment of surgical site infection. London: NICE.

National Institute for Health and Clinical Excellence. (2013) Surgical Site Infection. Evidence update June 2013. London: NICE.

World Health Organization. (2009a) WHO guidelines on hand hygiene in health care. Geneva: WHO.

World Health Organization. (2009b) WHO guidelines for safe surgery. Geneva: WHO.

World Health Organization. (2016) Global Guidelines for the Prevention of Surgical Site Infection. Geneva: WHO. 